\title{
Fabrication of Air Freshening Spongy Three Dimensional Electrospun Membrane
}

\author{
Purnima Mulmia, Hem Raj Pant ${ }^{\mathrm{a}, \mathrm{b}^{*}}$ \\ ${ }^{a}$ Dept. of Engineering Science \& Humanities, Pulchowk Campus, Institute of Engineering, TU, Nepal

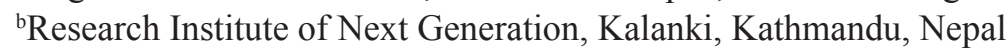 \\ *Corresponding author: hempant@ioe.edu.np
}

Received: Jan 8, 2018 Revised: Feb 12, $2018 \quad$ Accepted: Feb 15, 2018

\begin{abstract}
In this work, air freshening spongey 3D nanofibrous membrane of nylon-6 (N6) is fabricated via electrospinning process. Viscous N6 solution dissolved in formic acid/acetic acid solvent system was allowed for electrospinning to obtain 2D membrane. As-fabricated 2D membrane is converted into 3D one by using gas foaming technique. Lemon grass essential oil as a fragrance was incorporated through 2D and 3D porous membrane by evaporating essential oil at $60^{\circ} \mathrm{C}$ for 5 hours. The morphology of different membranes was examined from FE-SEM images which showed that 3D membrane is far porous than 2D one. The control fragrance release from the 3D spongy membrane was evaluated from the weight loss of oil-loaded membrane for 60 days which showed that the 3D membrane had more oil uptake and the controlled release of oil over the studied time. FT-IR spectra of oil-incorporated 3D membrane at different interval of times indicated the presence of fragrance even after the 60 days. The result showed that as-fabricated sponge 3D membrane may be a potential candidate for the future air freshening materials.
\end{abstract}

Keywords: Electrospinning, nanofibers, sodium borohydride, gas foaming technique, air fresheners

\section{Introduction}

Air fresheners are the consumer products that emit fragrance in order to create a pleasant smelling and clean indoor atmosphere at homes and offices. They contain different natural and synthetic compounds that deliver fragrance using different methods. Most of the air fresheners contain five basic ingredients: formaldehyde, phthalate, parabens, petroleum distillates, and p-dichlorobenzene that impose serious health hazards like nausea, infertility, neurological dysfunction, leukemia and cancer [13]. Many air fresheners do not even disclose their chemical constituents [12]. Few air fresheners utilize natural herbs and essential oils, but they require an active source like electric power to heat them up for fragrance delivery as in case of plugins [3].

There are varieties of fragrance carrier systems found in market. One of them is the use of nanofibrous mesh of non-toxic and degradable polymeric materials [7, 4]. The nano-fibrous mesh has a good capacity to uptake different liquid and vapor materials. Nanofibrous membrane can be 
fabricated by different process including template synthesis, drawing, phase-separation, selfassembly, electrospinning, etc. Out of them electrospinning is the versatile technique to fabricate continuous smooth nanofibers of variety of polymers [8-10]. However, membrane obtained from this process is $2 \mathrm{D}$ sheet-like structure which may not have sufficient empty space for foreign material uptake. Therefore, it is essential to convert 2D membrane into 3D one. Different postelectrospinning processes are reported for the conversion of 2D membrane into 3D one. Recently, Joshi et.al. proposed a versatile gas foaming technique to convert 2D electrospun membrane into 3D sponge [5]. For this, viscous polymer solution is prepared and nanofiber membrane is fabricated by passing this solution through strong electric field. As-fabricated membrane is then treated with gas foaming reagent to convert 2D membrane into $3 \mathrm{D}$ one containing large empty space inside the membrane [5]. Such 3D structure has found to be potential application in health care technology $[4,5]$. However, there is no any report about the use of such 3D electrospun membrane for fragrance delivery systems. Our concern is to upload Nepalese origin essential oil through such 3D membrane and study its control fragrance release capacity which can be effectively used for air freshening system for a long period of time. Since, natural essential oils as well as biocompatible polymer have no negative effects in human health; we except that asfabricated materials with control fragrance release capacity might be the potential candidate for future air freshening materials.

\section{Experimental}

\subsection{Materials}

Electrospun mat was fabricated from Nylon-6 $($ N6, Molecular weight $=35000)$ pellets obtained from Kolon, Korea. Formic acid and acetic acid were purchased from Anbaxy Fine Chemicals, India and Thermo Fisher Scientific, India, respectively. Sodium borohydride (SB) was obtained from Thomas Baker Chemicals, India while the lemongrass essential oil was purchased from Jadibuti Association of Nepal. All the chemicals were used without further purification.

\subsection{Electrospinning Setup and Procedure}

An N6 solution (22 wt\%) was prepared by dissolving N6 pellets in a solvent system of formic acid and acetic acid $(4: 1 \mathrm{w} / \mathrm{w})$ under magnetic stirring for $12 \mathrm{~h}$ at room temperature [4]. The electrospinning solution was drawn into plastic syringe and flow rate was maintained at $0.5 \mathrm{ml} / \mathrm{h}$. Electrospinning was performed at room conditions where the parameters include applied voltage of $10 \mathrm{kV}$, tip to collector distance of $20 \mathrm{~cm}$ and collector drum rotation speed of $500 \mathrm{rpm}$.

\subsection{Post Electrospinning Procedure}

The electrospun 2D mat of size $5 \mathrm{~cm} \times 5 \mathrm{~cm}$ (Fig. 1(a)) was transformed to a three dimensional spongy structure using SB solution as gas foaming reagent [10]. In order to obtain the desired structure of the 3D sponge, the 2D mat was soaked into the SB solution for varying amount of time (5 minutes, 30 minutes, 2 hours and 4 hours) and shaken continuously at $90 \mathrm{rpm}$ using a rotator. The SB solution was also varied at $0.1 \mathrm{M}, 0.5 \mathrm{M}$ and $1 \mathrm{M}$ concentration. The resulting $3 \mathrm{D}$ mats were washed with distilled water and dried for 24 hours, which is shown in Fig. 1(b). 


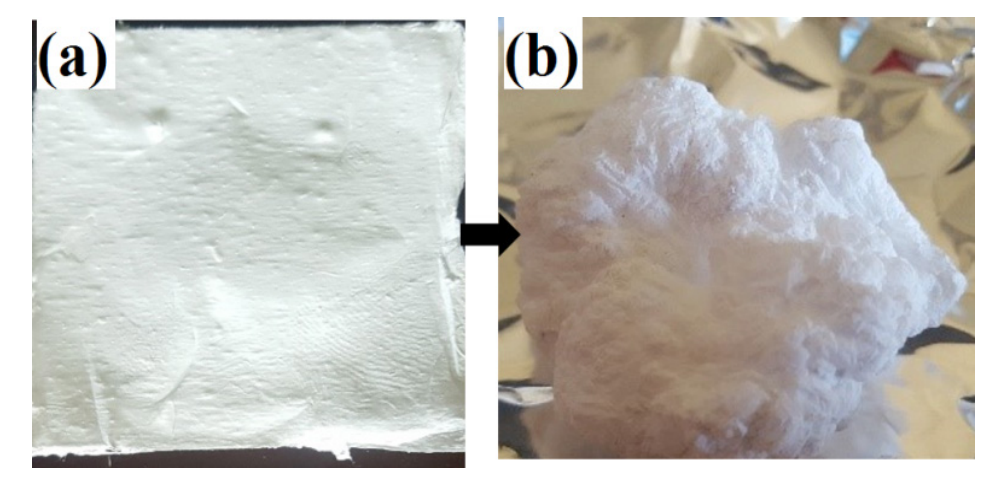

Fig.1. 2D electrospun N6 mat (a), and (b)3D electrospun sponge

\subsection{Addition of Fragrance Material}

Lemongrass oil was uploaded to the 2D and 3D electrospun mesh (Fig. 2b) by evaporation of the oil in a closed container for 5 hours. The temperature of oil while heating was at around $60^{\circ} \mathrm{C}$. To observe the effectiveness of 3D structure for oil absorption and its control fragrance release, the oil was evaporated into a 2D electrospun mat of equal weight (Fig. 2a).

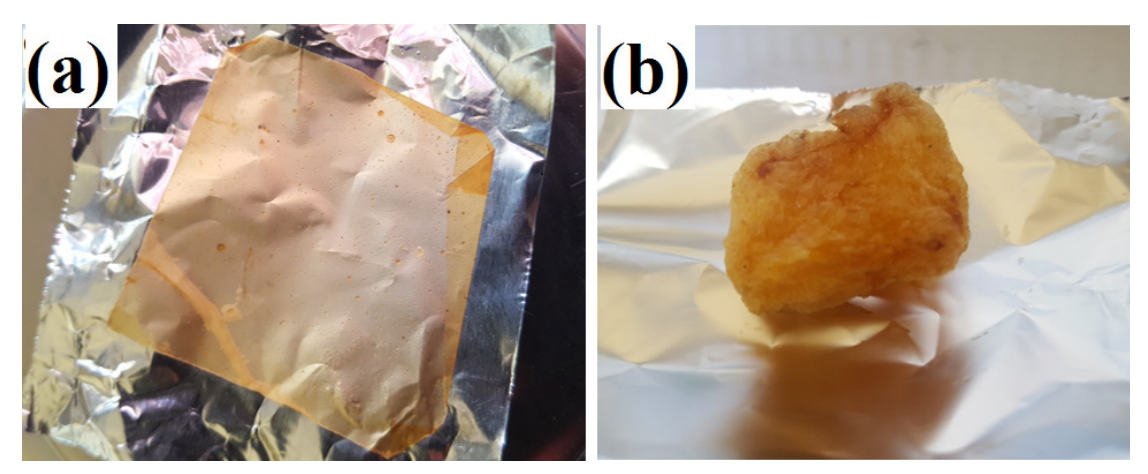

Fig. 2: Lemongrass oil added to (a) 2D electrospun N6 mat, and (b) 3D electrospun sponge

\section{Results and Discussion}

\subsection{Analysis of Gas Foaming Technique}

Treatment of 2D mats with SB solution resulted into 3D sponge due to hydrolysis reaction as shown in equation (1) liberating hydrogen gas bubbles that can easily push the fibers apart.

$$
\mathrm{NaBH}_{4}+\left(2+x \cdot \mathrm{H}_{2} \mathrm{O}\right)->\mathrm{NaBO}_{2} \cdot x \mathrm{H}_{2} \mathrm{O}+4 \mathrm{H}_{2}
$$

Higher dimension 3D sponges were obtained when the concentration of SB was $1 \mathrm{M}$ and the time of treatment was 4 hours as shown in Fig. 3. No significant change was seen in the 3D structure for time more than 4 hours. Treatment with SB for more than 12 hours caused damage to the structure which was observed during experiment. Therefore, the sponges treated for 4 hours were chosen as the optimum ones and further processing was carried out on them. 

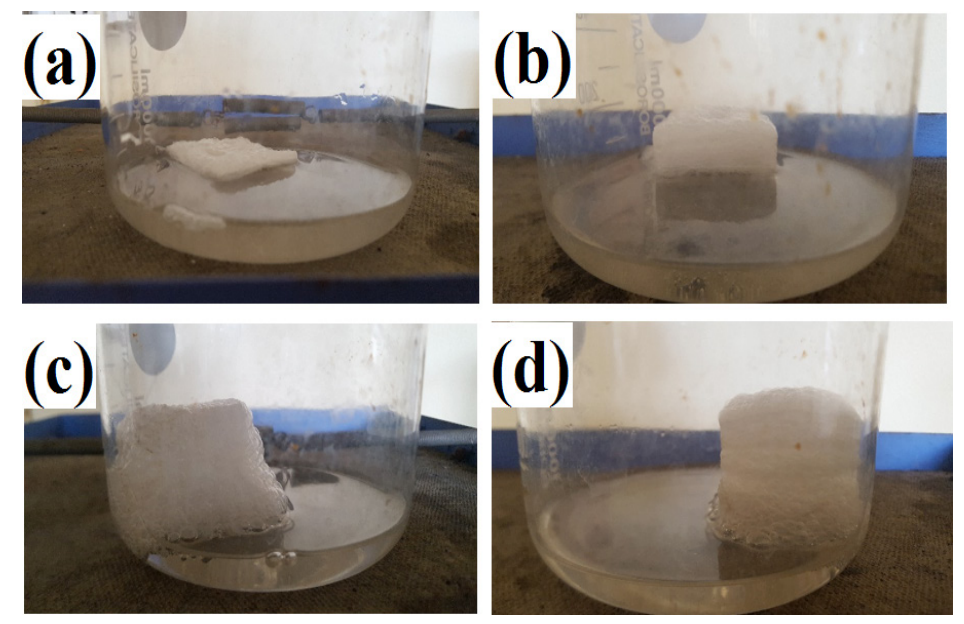

Fig. 3: Treatment of N6 fibrous mat with $1 \mathrm{M} \mathrm{SB}$ solution at $26^{\circ} \mathrm{C}$ for (a) $5 \mathrm{~min}$, (b) $30 \mathrm{~min}$, (c) 2 hrs (d) 4 hrs

\subsection{Morphology of Membrane}

The FE-SEM images of the pristine N6 2D mat and the 3D sponge under different magnification are shown in figure 4 . The diameter of the fibers were approximately $100-200 \mathrm{~nm}$. The image of 3D N6 showed an increase in the porosity of the structure when it was treated with SB solution. This is due to the hydrogen gas liberated in gas foaming technique using SB solution. The fibers were also more twisted and grouped together than pristine N6, which is due to the pressure exerted by gas molecules to the fibers. The FE-SEM image of the lemongrass added sponge under different magnification is shown in Fig. 5 (a), (b) and (c). The image shows a different morphology than that of pristine N6 mat. The higher magnification shows layered structure of 3D sponge with oil incorporated into it.

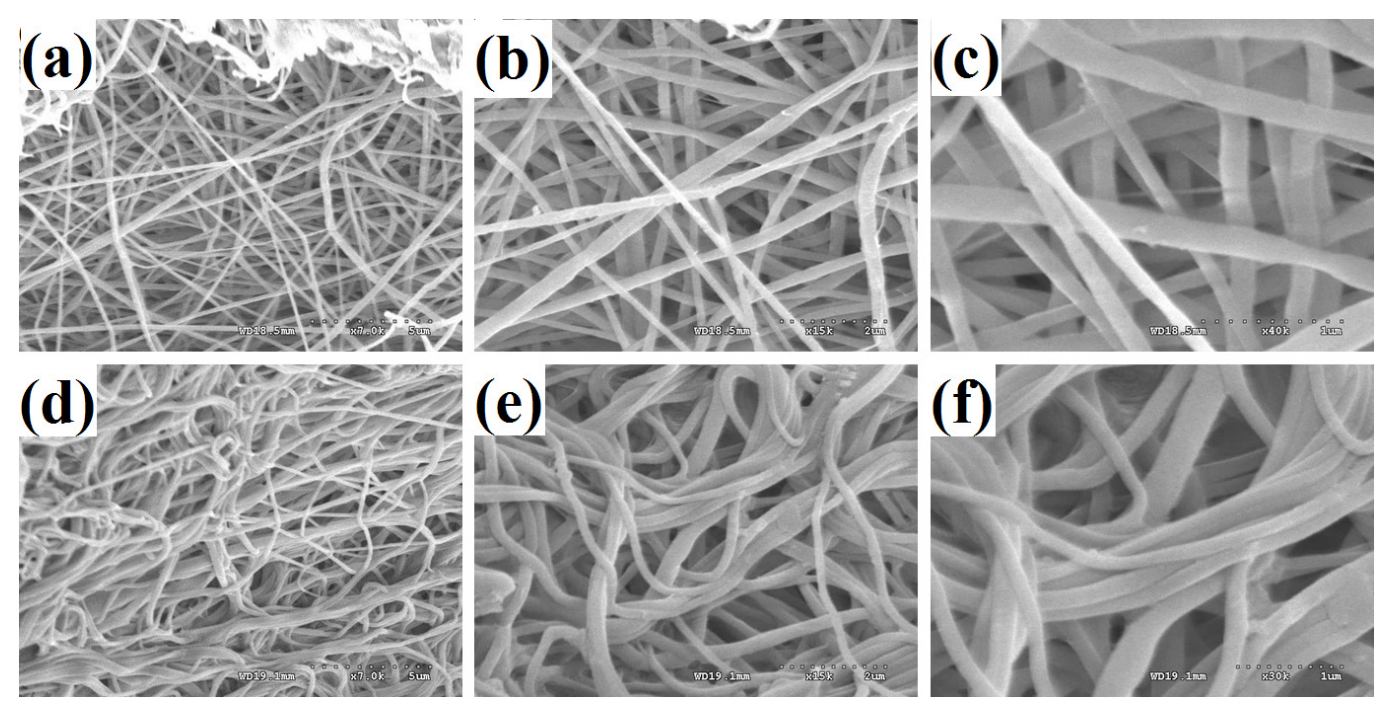

Fig. 4: FE-SEM images of pristine N6 mat under magnification (a) 7K (b)15K and (c) 40K; FE-SEM images of SB treated N6 mat under magnification (d) $7 \mathrm{~K}$ (e)15K and (f) $30 \mathrm{~K}$ 

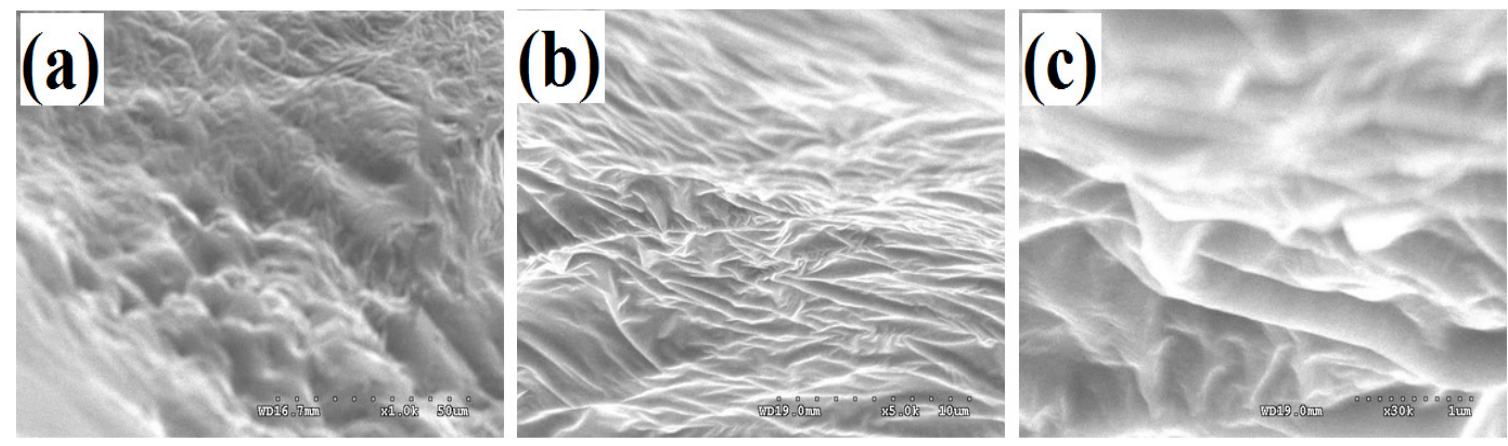

Fig. 5: FE-SEM images of oil added 3D N6 sponge under different magnification (a) $1 \mathrm{~K}$, (b) $5 \mathrm{~K}$ and (c) $30 \mathrm{~K}$

\subsection{Loading and Control Release of Fragrance}

After the addition of essential oil into equal weights of 2D mat and 3D mesh of N6, the weights of the samples were measured for a period of 60 days. During this period, there were negligible changes in the weights of 2D N6 mat and SB treated 3D sponge. However, there was significant change in the weight of oil-incorporated sample over this period. The decrease in weight of fragrance added 2D and 3D N6 structure over time is graphically shown in figure 6. During the first 3-4 days, there was a sharp decrease in the weight of both mats due to evaporation of the essential oils present near the surface. There was notable decrease in the weight for 3D mat over the duration of consideration corresponding to slower rate of evaporation of the oil from the mat while the weight of 2D mat remained constant after 6 days.

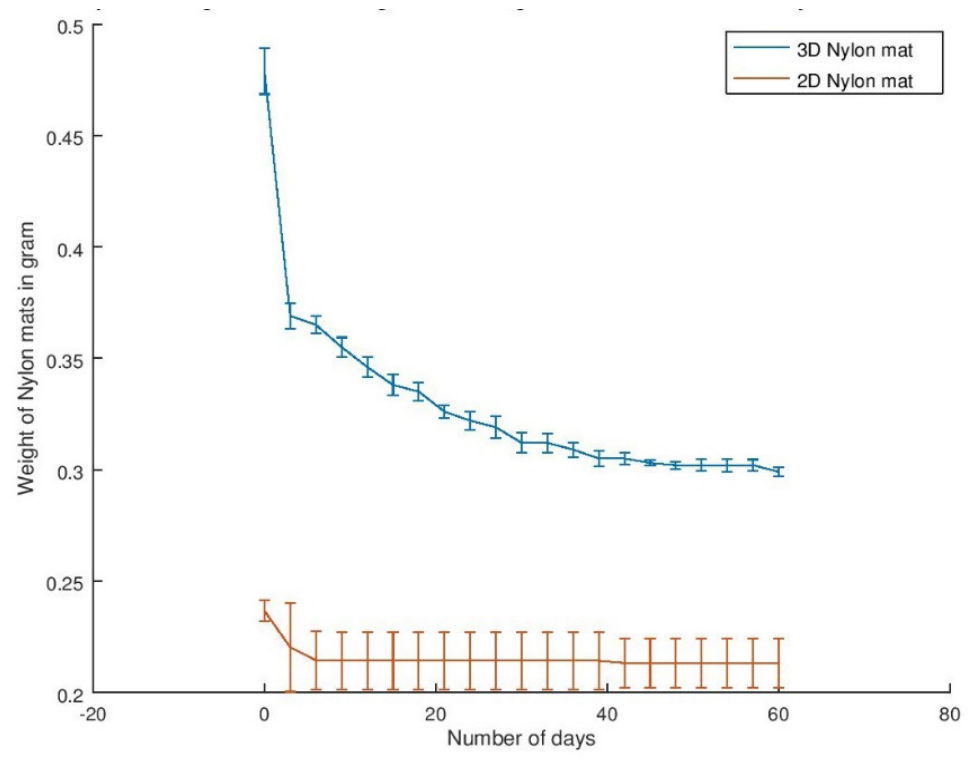

Fig. 6: Graph showing decrease in weights of lemongrass oil added N6 2D mat and 3D sponge during 60 days 
The porous structure of the nanofibrous electrospun sponge plays a significant role in uptake of essential oils. The oil deposited into the sponge by evaporation penetrates deep into it and slowly outer layers are filled with oil. The oil content in it volatilizes from the surfaces at the early period. As more surface area is exposed to the surrounding, more oil gets evaporated. After the oil at the surface is evaporated, there is a slow evaporation from successive layers. The decrease in weight of lemongrass only at room temperature over time and the oil content of the 3D sponge over same time was also studied. There was a sharp decrease in oil content of 3D sponge in early period but a slower rate of decrease was observed over time than the essential oil kept for vaporization at room temperature as shown in Fig. 7. The odor of 3D N6 sponge before and after oil incorporation was observed. The fragrance was still present in the structure for 60 days which indicates that the sponge can deliver fragrance for even longer period of time.

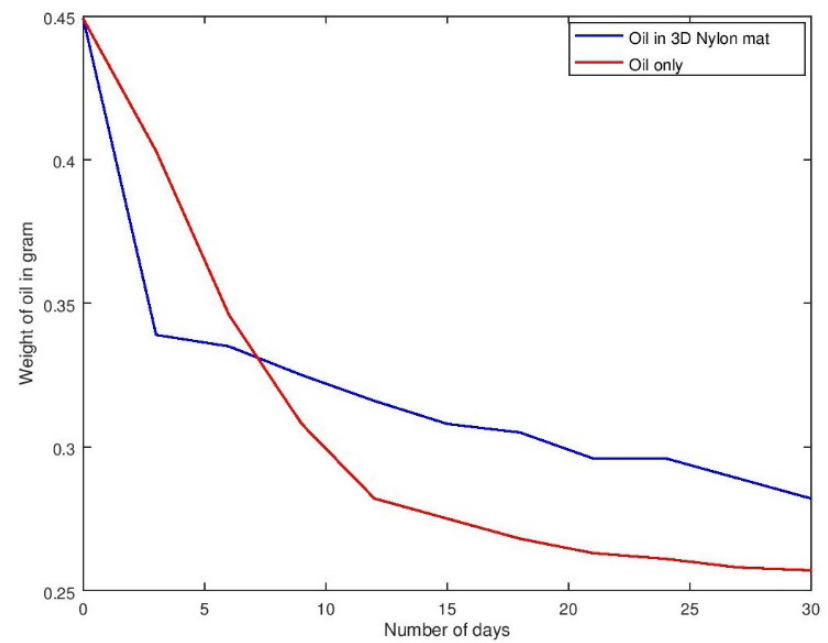

Fig. 7: Graph showing weight loss of free liquid lemongrass oil and oil loaded 3D sponge over time

\subsection{FTIR Analysis}

The FTIR spectra of the SB treated N6 sponge (Fig. 8) was similar to that of the pristine N6. Identical characteristic IR band of both pristine and SB treated N6 sponge indicated that there is no pronounced physiochemical changes in the fibers. The characteristic peaks in SB treated $\mathrm{N} 6$ are observed at $3300 \mathrm{~cm}^{-1}$ (corresponding to hydrogen bonding), $3180 \mathrm{~cm}^{-1}, 2950 \mathrm{~cm}^{-1}(\mathrm{CH} 2)$, $1640 \mathrm{~cm}^{-1}$ (Amide-I), $1540 \mathrm{~cm}^{-1}$ (Amide-II) and $1250 \mathrm{~cm}^{-1}[6,9]$. In case of lemongrass, the strong methylene/methyl band $\left(1446.5 \mathrm{~cm}^{-1}\right)$ and a weak methyl band $\left(1384.8 \mathrm{~cm}^{-1}\right)$, plus a band at 740 $\mathrm{cm}^{-1}$ (methylene rocking vibration) indicate a long chain linear aliphatic structure [7]. The saturated hydrocarbon $\mathrm{C}-\mathrm{H}$ stretching absorptions occur below $3000 \mathrm{~cm}^{-1}$. The band observed between 3150 and $3000 \mathrm{~cm}^{-1}$ are indicative of unsaturation $(\mathrm{C}=\mathrm{C}-\mathrm{H})$ and/or aromatic rings and their absence in the IR spectrograph obtained shows the absence of aromatic compounds. Carbonyl compounds lie between 1825 and $1575 \mathrm{~cm}^{-1}$ [13]. A band of 2850-2960 (alkyl group) is also present in the spectra. Similar peaks were observed in the N6 3D sponge added with lemongrass and the sponge treated with SB with a slight shift in the peaks was also observed. This may be due to the interaction between the functional groups of N6 and essential oil. Additional bands as compared to SB treated sponge shows that large amount of oil is loaded into the 3D sponge. The transmittance peak intensity for band between $3100-3500 \mathrm{~cm}^{-1}$ (corresponding to hydroxyl group) increased in oil added sponge than SB treated sponge. This may be due to the replacement of water content by oil 
or deposition of oil covering water molecules. FTIR spectra of oil added N6 sponge after 60 days also showed the presence of oil content in the membrane as shown in Fig. 9.

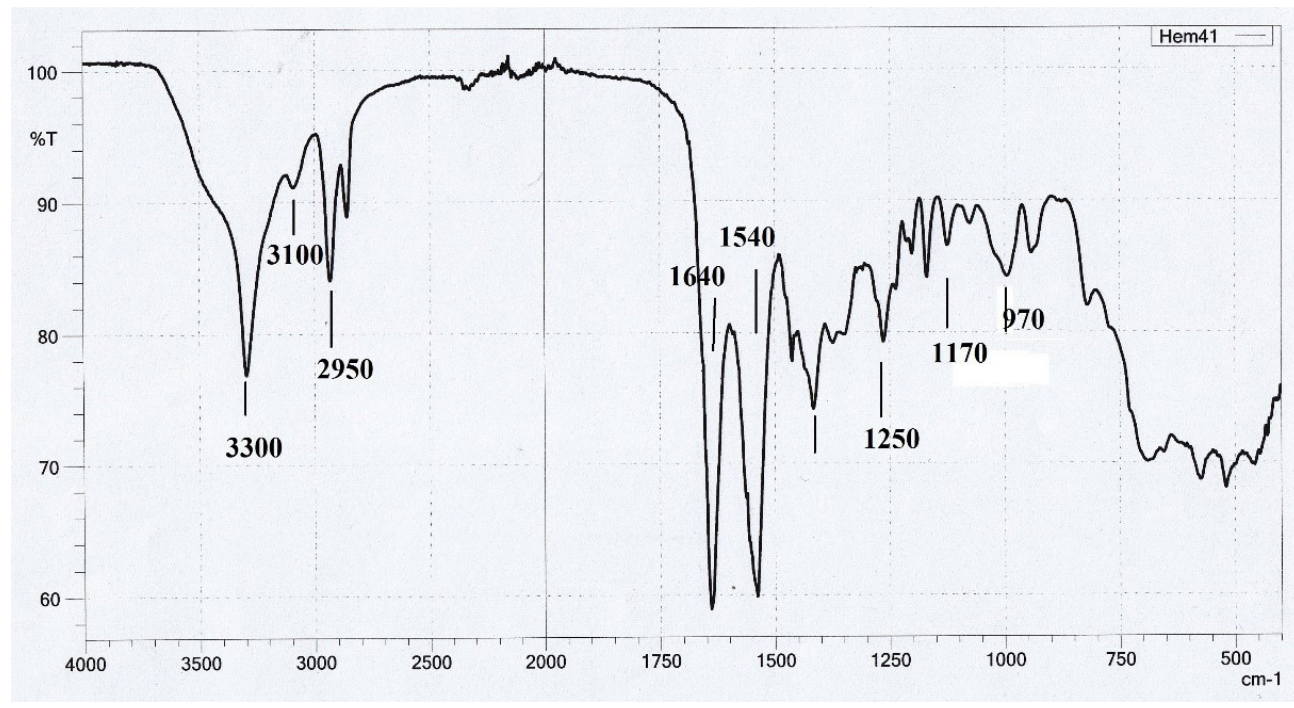

Fig. 8: FTIR spectra of N6 membrane treated with SB solution

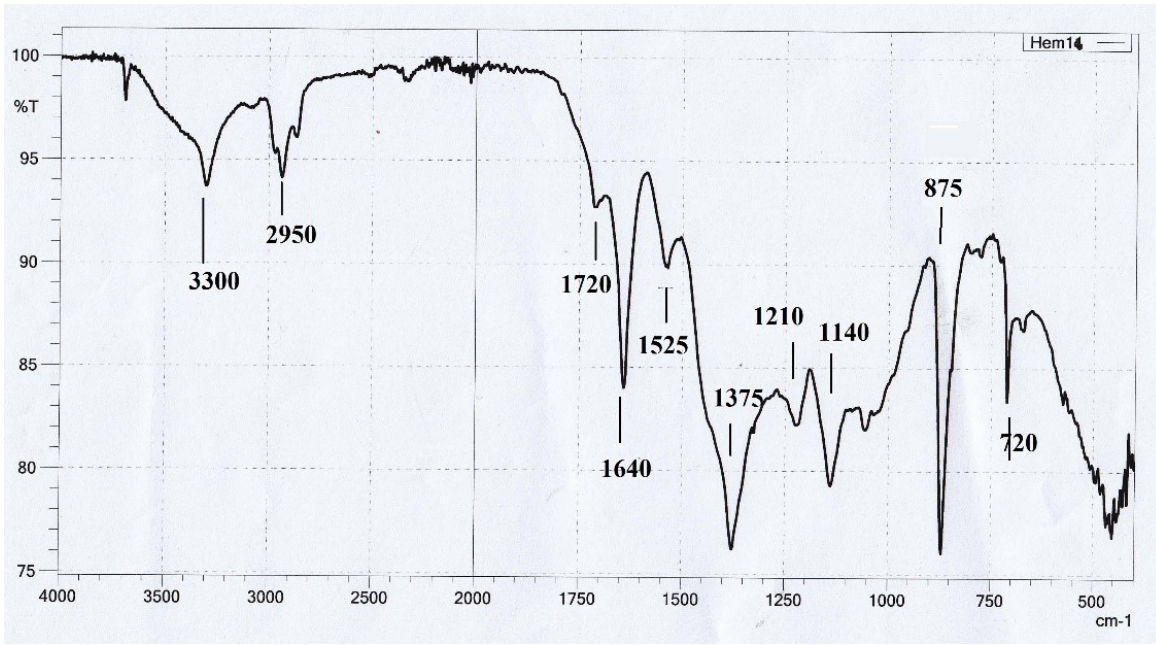

Fig. 9: FTIR spectra of lemongrass loaded 3D N6 ma

\section{Conclusions}

A 3D spongy membrane was fabricated from a conventional 2D electrospun N6 mat using facile gas foaming technique which provides large empty space for fragrance loading and its control release. Electrospun N6 nanofiber is used as fragrance carrier system owing to its non-toxicity, low degradability, ability to electrospin and ease of availability. Adding the oil to the N6 sponge slowed the rate of volatilization than the rate of oil alone, which makes it effective to be used as air freshener system. The 3D structure was able to uptake more essential oil than its $2 \mathrm{D}$ weight equivalent. This is due to the increase in pore size when treated with SB solution. Oil added 3D N6 
sponge showed layered structure i.e. pores filled with essential oil. Furthermore, the FTIR analysis confirmed the presence of lemongrass in 3D electrospun N6 sponge even after 60 days which shows that the sponge may able to release fragrance for further time also. Therefore, as-fabricated membrane can be used as control fragrance delivery system for future air freshening materials.

Acknowledgments: We are thankful to Chonbuk National University and Sogang University, Republic of Korea for the FE-SEM images, and Central Department of Chemistry, Tribhuwan University for FTIR spectroscopic analysis. We would also like to thank Robotics Club, Pulchowk Campus for their technical support for making evaporating set-up.

\section{References}

[1] Ambrosio L (2009), Biomedical composites. Elsevier.

[2] Bibiana MA, Selvamani P and Latha S (2012), In-vitro antimicrobial evaluation of extracts, oil and fractionated geraniol of cymbopogan citratus-an aromatic grass. International Journal of Environmental Sciences, 3(1) : 583-590.

[3] He MP, Triplett C and Conway MJ (2005), Wall-mounted electrical device having adjustable outlet prongs, US Patent 6, 897, 381

[4] Joshi MK, Pant HR, Tiwari AP, Maharjan B, Liao N, Park CH, Kim CS et al. (2016), Threedimensional cellulose sponge: Fabrication, characterization, biomimetic mineralization, and in vitro cell infiltration. Carbohydrate polymers, $136: 154-162$.

[5] Joshi MK, Pant HR, Tiwari AP, Park CH and Kim CS (2015), Multi-layered macroporous threedimensional nanofibrous scaffold via a novel gas foaming technique. Chemical Engineering Journal, 275 : 79-88.

[6] Lee KH, Kim KW, Pesapane A, Kim HY and Rabolt JF (2008), Polarized ft-ir study of macroscopically oriented electrospun nylon-6 nanofibers. Macromolecules, 41(4) : 1494-1498.

[7] Madivoli ES, Gitu L and Gumba E (2012), Isolation and identification of essential oils from cymbopogan citratus (stapf) dc using gc-ms and ft-ir. Chemistry and Materials Research, 2(4) : $13-22$.

[8] Pant HR, Pandeya DR, Nam KT, Baek WI, Hong ST and Kim HY (2011), Photocatalytic and antibacterial properties of a $\mathrm{TiO}_{2}$ /nylon-6 electrospun nanocomposite mat containing silver nanoparticles. Journal of Hazardous Materials, 189 : 465-471.

[9] Pant HR, Bajgai MP, Yi CA, Nirmala R, Nam KT, Baek WI, et al (2011). Effect of successive electrospinning and the strength of hydrogen bond on the morphology of electrospun nylon-6 nanofibers. Colloid Surface A, 370 : 87-94.

[10] Pant HR, Pant B, Park CH, Kim HJ, Lee DS, Tijing LD, et al. (2013), RGO/Nylon-6 Composite Mat with Unique Structural Features and Electrical Properties Obtained from Electrospinning and Hydrothermal Process. Fibers and Polymers, 14 : 970-975.

[11] Poursamar SA, Hatami J, Lehner AN, da Silva CL, Ferreira FC, Paula A and Antunes M (2015), Gelatin porous scaffolds fabricated using a modified gas foaming technique: characterisation and cytotoxicity assessment. Materials Science and engineering: $C, 48: 63-70$.

[12] Steinemann AC (2009), Fragranced consumer products and undisclosed ingredients. Environmental Impact Assessment Review, 29(1) : 32-38.

[13] Trantallidi M, Dimitroulopoulou C, Wolkoff P, Kephalopoulos S and Carrer P (2015), Ephect iii: Health risk assessment of exposure to household consumer products. Science of The Total Environment, 536 : 903-913. 